\title{
Cervical cancer \\ prevention: Review \\ of the past, present, \\ and future
}

\section{By Aimee Chism Holland, DNP, WHNP-BC, FNP-C, FAANP, and Kim Choma, DNP, APN, WHNP-BC}

The purpose of this article is to summarize the history of cervical cancer research supporting current practice guidelines used today. Recent updates to the human papillomavirus vaccination schedule, in addition to the latest cervical cancer screening and management guidelines, are highlighted. Future changes on the horizon are also discussed.

KEY WORDS: cervical cancer screening, human papillomavirus vaccine, cervical intraepithelial neoplasia, consensus guidelines

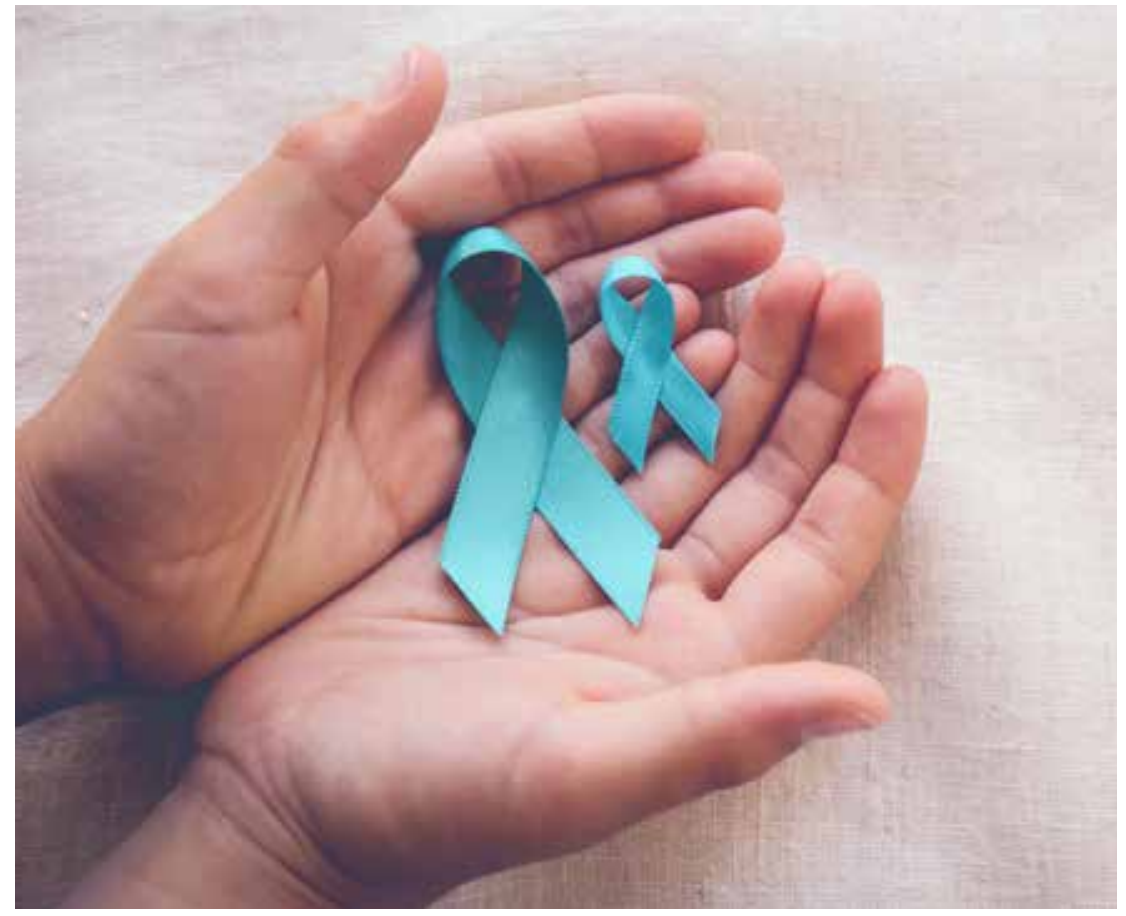

hree sets of recommendations that help prevent cervical cancer have recently been updated in the United States. The first two are the cervical cancer screening and management guidelines from the 2019 American Society of Colposcopy and Cervical Pathology (ASCCP) Risk-Based Management Consensus Guidelines and the 2020 American Cancer Society (ACS) cervical cancer screening guidelines for individuals at average risk. ${ }^{1,2}$ The third update is that of the vaccination schedule for the human papillomavirus (HPV) vaccine. ${ }^{3,4}$ Knowledge about the historical perspective of cervical cancer screening and management guidelines can help clinicians understand current guidelines and potential updates in the future. The purpose of this article is to inform clinicians about these most currently published cervical cancer screening and management guidelines while also providing them with an historical overview of the evolution of research supporting cervical cancer prevention.

\section{Historical background}

Cervical cancer is the fourth most common cancer among women globally. ${ }^{5}$ Approximately 311,000 deaths attributed to cervical cancer 
occur annually around the world, with more than $85 \%$ occurring in undeveloped countries. ${ }^{6}$ Cervical cancer was once the most common cause of US gynecologic cancer deaths. ${ }^{7}$ This is no longer the case due largely to research developments initially led by Dr. George Papanicolau in the early 1940s.

Papanicolau, a renowned pathologist at New York Hospital, discovered that swabbing the cervix for cells could demonstrate a difference between normal and abnormal cells as viewed under a microscope. ${ }^{8} \mathrm{His}$ pioneering work led to the development of the Pap smear. Today, cervical cytology is performed using a liquid base and is referred to as the Pap test. Papanicolau's research contributions led to a decline in US cervical cancer cases. ${ }^{8}$

HPV 16 and 18 were first detected in cervical cancer cells in the early 1980s. Epidemiologic studies followed this discovery demonstrating a consistent association between HPV and cervical cancer and the establishment by 1999 that HPV is the major cause of cervical cancer. ${ }^{9}$ The first HPV vaccination was US Food and Drug Administration (FDA) approved in $2006 .{ }^{10} \mathrm{It}$ is a quadrivalent HPV vaccine that targets types 6,11 , 16 , and 18 for males and females ages 9 to 26 years. HPV 16 and 18 are high-risk strands accountable for $70 \%$ of cervical cancers and precancerous cervical lesions. HPV 6 and 11 are low-risk strands responsible for anogenital warts. ${ }^{10} \mathrm{~A}$ bivalent vaccine that targets types 16 and 18 was FDA approved from 2009 until 2016 for females between the ages of 10 and 25 years. ${ }^{10}$ In all, three different vaccines have been developed and approved by the FDA. The most recent is the 9-valent vaccine, currently the only one available in this country, which was FDA approved in 2014.

\section{Present: Cervical cancer screening and HPV vaccination}

Today in the United States and other developed countries, cervical cancer screening and management guidelines, along with HPV vaccination guidelines, are in place for the prevention of persistent high-risk HPV infections and cervical cancer. Cervical cancer screening provides the opportunity for precancerous cervical lesions to be identified and treated early before cancer develops. Organized screening programs have demonstrated that early treatment of precancerous lesions prevents up to $80 \%$ of cervical cancers. ${ }^{6}$ Nevertheless, we continue to see cases diagnosed every year due to a lack of access to routine screening and loss to follow-up after abnormal screening results. ${ }^{11}$ HPV vaccination can prevent the most common HPV types associated with cervical cancer, genital warts, and other cancers of the vulva, vagina, anus, and oropharynx. ${ }^{12}$ Yet current HPV vaccination rates are less than desired. No longer should anyone living in the United States die from cervical cancer because this cancer is preventable. ${ }^{13}$

Twelve high-risk types of HPV $(16,18,31,33,35,39,45,51,52,56$, $58,59)$ are classified as carcinogenic by the International Agency for Research on Cancer. ${ }^{16}$ High-risk types of HPV cause $90 \%$ of cervical cancer. ${ }^{11,17}$ The majority of HPV-related cervical infections resolve within 24 to 36 months. ${ }^{10,18}$ Persistent high-risk HPV infections lead to precancerous lesions responsible for a diagnosis of cervical intraepithelial neoplasia (CIN). ${ }^{19}$ Precancerous lesions that have not advanced yet to CIN3 often regress spontaneously, but these can also progress to invasive cervical cancer if left untreated. The average time it takes a CIN3 lesion to progress to cervical cancer is 10 years. ${ }^{17}$ Therefore, screening and treatment of persistent high-risk HPV infections provide an opportunity for prevention of cervical cancer.

Clinicians should follow current evidence-based recommendations for cervical cancer screening for individuals with average risk endorsed by the American College of Obstetricians and Gynecologists (ACOG), ASCCP, the United States Preventive Services Task Force (USPSTF), and the ACS. 2,14,15 Individuals at high risk for cervical cancer should follow screening guidelines specific to their condition. Individuals at higher than average risk are defined as anyone with a suppressed immune system from human immunodeficiency virus infection, organ transplant, long-term steroid treatment, or an individual exposed to diethylstilbestrol in utero, and those previously treated for CIN grade 2 or higher. ${ }^{2}$

$H P V$ vaccination is most effective at preventing cervical cancer if received before HPV exposure, but it is still recommended after sexual debut. ${ }^{9}$ The vaccine does not eliminate existing HPV infections or related diseases. ${ }^{9}$ Current studies that have followed vaccinated individuals for 14 years suggest that sufficient protection from HPV continues long term and individuals do not need to be revaccinated. ${ }^{20}$ It is important, however, for vaccinated individuals with a cervix to continue routine cervical cancer screening because vaccination does not protect against already existing HPV infection nor does it cover all HPV types.

As mentioned previously, the 9-valent HPV vaccine approved by the FDA in 2014 is the only one now available in the United States. The 9-valent HPV vaccine protects against the same four HPV types 6, 11,16 , and 18 as the quadrivalent vaccine in addition to five other 
high-risk oncogenic types including $31,33,45,52$, and $58 .{ }^{21}$ Clinicians should follow the HPV vaccine schedule recommended by the Centers for Disease Control and Prevention (CDC). The vaccine has been approved by the FDA for individuals up to age 45 years. The CDC guidelines routinely recommend it for individuals up to age 26 years, however, because it provides fewer benefits to individuals who have already been exposed to HPV. ${ }^{22}$

Two doses of the 9-valent HPV vaccine series are recommended by the CDC at age 11 or 12 years and up to age 14 years, but these can be initiated as early as at age 9 years. ${ }^{22}$ Three doses are recommended if the initial dose is received between ages 15 and 26 years. ${ }^{22}$ If an individual and their healthcare provider determine to vaccinate after age 26 years, then three doses should be received. ${ }^{22}$ Shared decision making is recommended for the clinician and patient to ascertain if $\mathrm{HPV}$ vaccination is needed after the age of 26 years. ${ }^{22}$

\section{Present: Highlights from the 2019 ASCCP Risk-Based Management Consensus Guidelines}

The 2019 ASCCP Risk-Based Management Consensus Guidelines for abnormal cervical cancer screening tests and cancer precursors marks the fourth set of management guidelines published since the original consensus conference in 2001. ${ }^{1}$ This document replaces previously published guidelines released in 2001, 2006, and 2012 for managing patients with abnormal cervical cancer screening results. ${ }^{23-25}$ The consensus process was a 2-year collaboration of 19 organizations, including professional medical societies, federal agencies, and patient advocacy groups. The process moved guidelines away from management algorithms based on test results and developed a framework for managing patients based on their risk of currently having or developing high-grade precancer, defined as CIN3 or higher (CIN3+). The guidelines establish risk thresholds that correspond to different clinical actions. The estimation of risks of CIN3+ are based on the combination of a patient's current cervical cancer screening results and past results. ${ }^{1}$ First, immediate CIN3+ risk is determined. If immediate risk is $4 \%$ or higher, clinical actions will fall into the categories of colposcopy or expedited treatment depending on how high the immediate risk is estimated to be. ${ }^{1}$ For patients at highest risk, defined as a $60 \%$ risk or higher of currently having CIN3+, expedited treatment proceeding directly to excisional treatment without first performing a colposcopic biopsy is preferred. ${ }^{1}$ Patients with a current risk of CIN3+ between $25 \%$ and $59 \%$ can choose between expedited treatment and colposcopy. ${ }^{1}$ Colposcopy is preferred for patients whose current risk of CIN3+ is $4 \%$ to $24 \%$. $^{1}$ Patients with an immediate $\mathrm{CIN} 3+$ risk less than $4 \%$ fall below the colposcopy threshold. ${ }^{1}$ For these patients, surveillance with repeat $H P V$ testing or cotesting at 1,3 , or 5 years is recommended with intervals determined by estimated 5-year CIN3+ risk. ${ }^{1}$

ASCCP recognizes that the 2019 Risk-Based Management Consensus Guidelines include a number of changes. Therefore, a new app and web application system was created to help clinicians streamline the data. ${ }^{26}$ Following is a scenario demonstrating decision making based on patient data and use of the ASCCP app.

\section{Case study scenario}

A 26-year-old patient receives an atypical squamous cells of unde- termined significance (ASCUS) Pap and a positive HPV 16 test on her recent screen (Photos 1,2). Previous screening results at age 22 years were ASCUS/negative HPV (Photo 3). Based on the 2019 Risk-Based Management Consensus Guidelines, what should the clinician do next?

Based on the new ASCCP app, the clinician should perform HPVbased screening in 12 months. ${ }^{27}$ No treatment is needed at this time because an individual with these results and with this history only has a 6.6\% 5-year risk of developing CIN3+ (Photo 4). ${ }^{1,27}$

\section{Summary of changes from previous guidelines}

These are the primary changes from prior management guidelines:

- Recommendations for colposcopy, treatment, or surveillance are based on a patient's risk of $\mathrm{CIN} 3+{ }^{1}$ A patient's risk of developing $\mathrm{CIN} 3+$ is determined by their current screening results in addition to their history of past test results. ${ }^{1}$ The longer an HPV infection is present, the higher the risk for developing precancer and cancer. ${ }^{1}$

- Certain patients with minor screening abnormalities indicative of HPV infection with low risk of underlying $\mathrm{CIN} 3+$ may not initially need a colposcopy but instead may need a repeat HPV test or cotest in 1 year. ${ }^{1}$

- Screening with HPV testing is recommended, but when $\mathrm{HPV}$ testing or cotesting is not available, then cytology alone is acceptable. ${ }^{1}$ Cytology is less sensitive than HPV testing for detecting precancer, so shorter intervals for testing are needed. ${ }^{1}$

- Colposcopy is recommended for patients with an immediate 


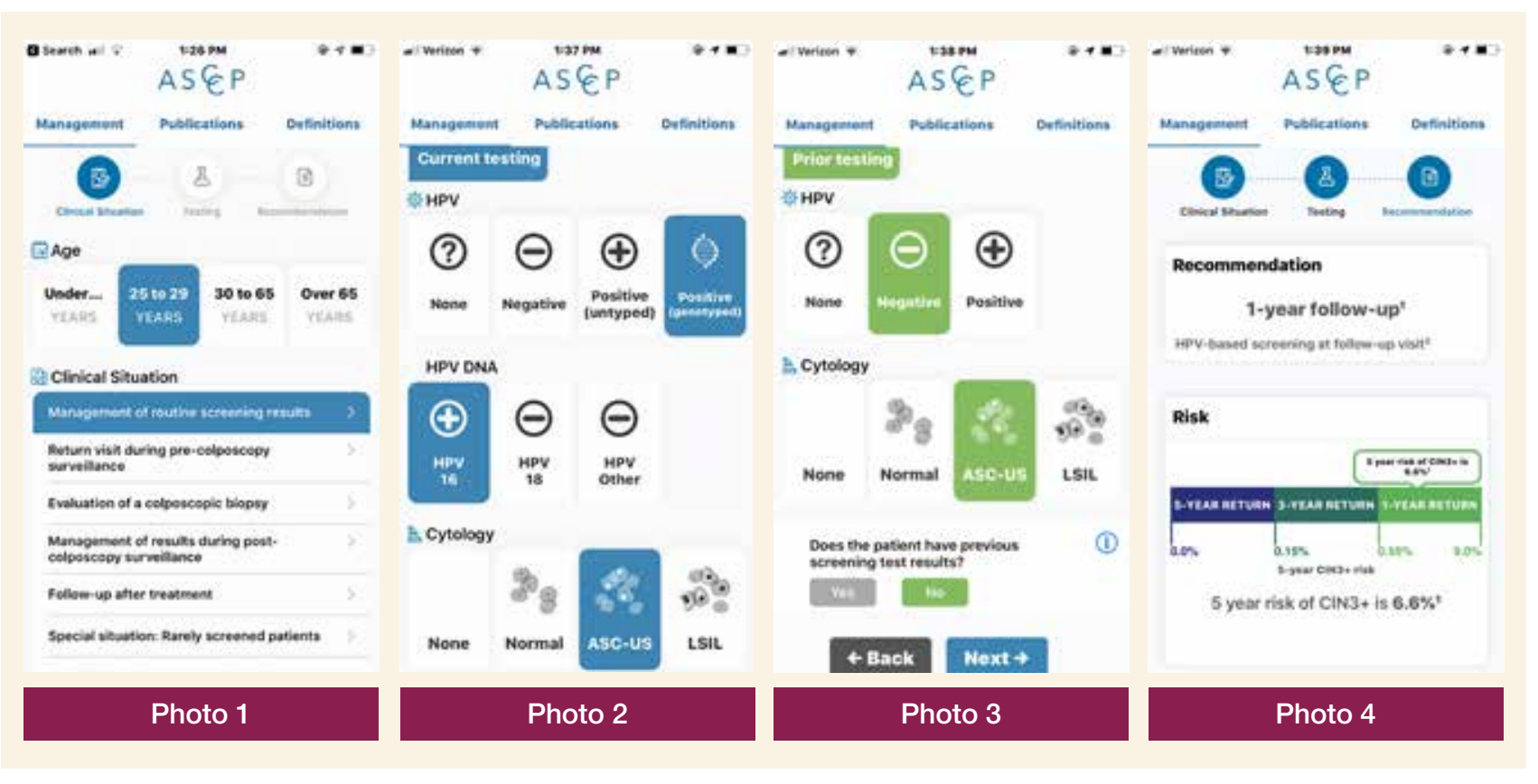

risk of $4 \%$ or greater for developing CIN3+ based on prior history and current results. ${ }^{1}$

- Patients at high risk should receive expedited treatment. ${ }^{1}$ Expedited treatment guidance has been expanded and should include shared decision making, especially for patients who have concerns about pregnancy outcomes secondary to treatment. $^{1}$

- Excisional treatment is preferred to ablative treatment for CIN2 or CIN3 and is recommended for adenocarcinoma in situ (AIS). ${ }^{1}$

- Observation is preferred for CIN1 to avoid unnecessary procedures in low-risk patients. ${ }^{1}$ Treatment is acceptable for patients with persistent CIN1 for 2 years or longer. ${ }^{1}$

- All positive primary HPV screening results should have reflex testing to include both genotyping if not already done and cytology from the same laboratory specimen because the findings can inform colposcopy practice. ${ }^{1}$ If additional lab- oratory testing is not possible when HPV 16 or HPV 18 testing is positive, then colposcopy should be performed. ${ }^{1}$

- After the treatment and initial post-treatment management of histologic HSIL [high-grade squamous intraepithelial lesion], CIN2, CIN3, or AIS, surveillance with HPV testing or cotesting should be continued at 3-year intervals for a minimum of 25 years. ${ }^{1}$

- Risk-based thresholds are now defined for follow-up intervals of 1-, 3-, and 5-year return for patients whose risk of $\mathrm{CIN} 3+$ is too high for routine screening but not high enough to warrant colposcopy.

- Establishment of risk-based thresholds provides a way for new tests to be evaluated against existing thresholds, thus accommodating new tests in development and reducing the need for frequent interim guidelines and recurrent consensus conferences. ${ }^{1}$

- Only FDA-approved HPV assays for screening high-risk HPV types should be used for management purposes. ${ }^{1}$

\section{Future: Guideline updates and new technology}

Recognizing that many new tests for cervical cancer screening and management of abnormal screening test results are in development, the consensus organizations continue to work together in the Enduring Guidelines process. ${ }^{1}$ This committee of about 20 organizations is led by the National Cancer Institute, CDC, ACS, and ASCCP. Organizational representatives will meet on a continual basis to review data on new assays and technologies with regulatory approval and decide how they should be used within the framework of clinical action risk thresholds developed during the 2019 ASCCP Risk-Based Management Consensus Guidelines process.

In the past, as new tests were developed, new guidelines or interim guidance statements were issued. The Enduring Guidelines process allows new data to be readily incorporated into the existing guidelines 


\section{It is hopeful that in the near future we will have a therapeutic HPV vaccine.}

framework without initiating new consensus meetings. ${ }^{1}$ The establishment of risk-based thresholds means that new tests can be evaluated against existing thresholds instead of making new algorithms for each new test, and the new guidance can be easily incorporated into existing decision support tools. ${ }^{1}$ This process can also be used to evaluate when population-level changes, such as rising HPV vaccination rates, should be incorporated into management recommendations.

It is hopeful that in the near future we will have a therapeutic HPV vaccine. Therapeutic vaccines differ from prophylactic vaccines in that they stimulate cell-mediated immunity and kill infected cells. ${ }^{9}$ This type of vaccine has been developed, but it is still in clinical trials. ${ }^{9}$

\section{Conclusion}

This article provides updates to current cervical cancer prevention guidelines. Past, present, and future research supporting practice guidelines is summarized to help clinicians understand current guideline changes and potential updates in the future.

\section{Aimee Chism Holland is Associate Professor at the University of Al- abama at Birmingham School of Nursing, Birmingham, Alabama. Kim Choma is on the staff of Ob/ Gyn Associates of Northern $\mathrm{NJ}$ in}

Teaneck, New Jersey. The authors have no actual or potential conflicts of interest in relation to the contents of this article.

Acknowledgment: The authors would like to acknowledge Nicolas Wentzensen, MD, PhD, MS, and Rebecca B. Perkins, MD, MSc, for their contributions to this article.

\section{References}

1. Perkins RB, Guido RS, Castle PE, et al. 2019 ASCCP Risk-Based Management Consensus Guidelines for abnormal cervical cancer screening tests and cancer precursors. J Low Genit Tract Dis. 2020;24(2):102-131.

2. Fontham ETH, Wolf AMD, Church TR, et al. Cervical cancer screening for individuals at average risk: 2020 guideline update from the American Cancer Society. Ca Cancer J Clin. 2020;70(5):321-346.

3. MerckVaccines. Dosage and administration for Gardasil 9. 2020. https://www.merckvaccines.com/ gardasil9/dosing-administration/

4. Centers for Disease Control and Prevention. HPV vaccine schedule and dosing. August 15, 2019. https://www.cdc.gov/hpv/hcp/ schedules-recommendations.html.

5. Arbyn M, Weiderpass E, Bruni L, et al. Estimates of incidence and mortality of cervical cancer in 2018: a worldwide analysis. Lancet Glob Health. 2020;8(2):e191-e203.

6. World Health Organization. Human papillomavirus (HPV) and cervical cancer. November 11 , 2020. https://www.who.int/ news-room/fact-sheets/detail/human-papillomavirus-(hpv)-and-cervical-cancer.
7. Centers for Disease Control and Prevention. Cervical cancer statistics. June 8, 2020. https://www. cdc.gov/cancer/cervical/statistics/ index.htm.

8. Yong Tan S, Tatsumura Y. George Papanicolaou (1883-1962): discoverer of the Pap smear. Singapore Med J. 2015;56(10):586-587.

9. Wang R, Pan W, Jin L, et al Human papillomavirus vaccine against cervical cancer: opportunity and challenge. Cancer Lett. 2020;471:88-102

10. Insinga RP, Dasbach EJ, Elbasha $\mathrm{EH}$, et al. Incidence and duration of cervical human papillomavirus $6,11,16$, and 18 infections in young women: an evaluation from multiple analytic perspectives. Cancer Epidemiol Biomarkers Prev. 2007;16(4):709-715.

11. Melinkow J, Henderson JT, Burda $\mathrm{BU}$, et al. Screening for Cervical Cancer with High-Risk Human Papillomavirus Testing: A Systematic Evidence Review for the U.S Preventive Services Task Force. Evidence Synthesis No. 158. Rockville, MD: Agency for Healthcare Research and Quality; 2018.

12. Lei J, Ploner A, Elfstrom KM, et al. HPV vaccination and the risk of invasive cervical cancer. $N$ Engl J Med. 2020;383(14):1340-1348.

13. Centers for Disease Control and Prevention. Cervical cancer is preventable. January 6, 2020. https:// www.cdc.gov/vitalsigns/cervical-cancer/index.html.

14. ACOG Committee on Practice Bulletins-Gynecology. Practice

(continued on page 29) 
Obstet Gynecol. 2016:128(4):e111-e130.

28. US Preventive Services Task Force; Curry SJ, Krist AH, Owens DK, et al. Screening for cervical cancer: US Preventive Services Task Force recommendation statement. JAMA. 2018;320(7):674-686.

29. Fontham ETH, Wolf AMD, Church TR. Cervical cancer screening for individuals at average risk: 2020 guideline update from the American Cancer Society. CA Cancer J Clin. 2020;70(5):321-346.

30. Dinkelspiel H, Fetterman B, Poitras N, et al. Screening history preceding a diagnosis of cervical cancer in women age 65 and older. Gynecol Oncol. 2012;126(2):203-206.

31. White MC, Shoemaker ML, Benard VB. Cervical cancer screening and incidence by age: unmet needs near and after the stopping age for screening. Am J Prev Med. 2017;53(3):392-395.

32. Centers for Disease Control and Prevention. Increasing Population-based Breast and Cervical Cancer Screenings: An Action Guide to Facilitate Evidence-based Strategies. Atlanta, GA: Centers for Disease Control and Prevention, US Department of Health and Human Services; 2014. cdc.gov/ cancer/nbccedp/pdf/breastcanceractionguide.pdf. high-risk human papillomavirus testing for cervical cancer screening: interim clinical guidance. Gynecol Oncol. 2015;136(2):178-182.

34. Perkins RB, Guido RS, Castle PE, et al. 2019 ASCCP riskbased management consensus guidelines for abnormal cervical cancer screening tests and cancer precursors. J Low Genit Tract Dis. 2020;24(2):102-131.

\section{Web resources}

A. asccp.org/mobile-app

B. cdc.gov/cancer/nbccedp/index.htm

C. wypca.org/wp-content/uploads/breastcanceractionguide.pdf

D. npwh.org

E. hpvroundtable.org

Approved by the NPWH Board of Directors, March 2018.

Updated and reaffirmed by the Board of Directors, November 2020.

33. Huh WK, Ault KA, Chelmow D, et al. Use of primary

\section{(continued from page 20)}

bulletin no. 168: Cervical cancer screening and prevention. Obstet Gynecol. 2016:128(4):e111-e130.

15. US Preventive Services Task Force; Curry SJ, Krist AH, Owens DK, et al. Screening for cervical cancer: US Preventive Services Task Force recommendation statement. JAMA. 2018;320(7):674-686.

16. World Health Organization. Human Papillomaviruses. IARC Monographs on the Evaluation of Carcinogenic Risks to Humans, vol. 90. Lyon, France: World Health Organization; 2005.

17. Moscicki AB, Schiffman M, Burchell A, et al. Updating the natural history of human papillomavirus and anogenital cancers. Vaccine. 2012;30(suppl 5):F24-F33.

18. Plummer M, Schiffman M, Castle $\mathrm{PE}$, et al. A 2-year prospective study of human papillomavirus persistence among women with a cytological diagnosis of atypical squamous cells of undetermined significance or low-grade squa- mous intraepithelial lesion. J Infect Dis. 2007;195(11):1582-1589.

19. de Sanjose S, Brotons M, Pavon MA. The natural history of human papillomavirus infection. Best Prac Res Clin Obstet Gynaecol. 2018;47:2-13.

20. Kjaer SK, Nygard M, Sundstrom K, et al. Final analysis of a 14-year long-term follow-up study of the effectiveness and immunogenicity of the quadrivalent human papillomavirus vaccine in women from four nordic countries. EClinicalMedicine. 2020;23:100401.

21. Zhang Z, Zhang J, Xia N, Zhao Q. Expanded strain coverage for a highly successful public health tool: prophylactic 9-valent human papillomavirus vaccine. Hum Vaccin Immunother. 2017;13(10):2280-2291.

22. Centers for Disease Control and Prevention. HPV vaccine schedule and dosing. August 15, 2019. https://www.cdc.gov/hpv/hcp/ schedules-recommendations.html.

23. Wright TC Jr, Cox JT, Massad LS, et al. 2001 consensus guidelines for the management of women with cervical cytological abnormalities. J Low Genit Tract Dis. 2002;6(2):127-143.

24. Wright TC Jr, Massad LS, Dunton CJ, et al. 2006 consensus guidelines for the management of women with abnormal cervical cancer screening tests. Am J Obstet Gynecol. 2007;197(4):346-355.

25. Massad LS, Einstein MH, Huh WK, et al. 2012 updated consensus guidelines for the management of abnormal cervical cancer screening tests and cancer precursors. Obstet Gynecol. 2013;121(4):829846.

26. ASCCP. Mobile app. ASCCP RiskBased Management Consensus Guidelines. 2020. https://www. asccp.org/mobile-app.

27. Egemen D, Cheung LC, Chen X, et al. Risk estimates supporting the 2019 ASCCP Risk-Based Management Consensus Guidelines. J Low Genit Tract Dis. 2020;24(2):132143. 